\title{
Silicon micro-levers and a multilayer graphene membrane studied via laser photoacoustic detection
}

\author{
Z. Zelinger ${ }^{1}$, P. Janda ${ }^{1}$, J. Suchánek ${ }^{1,2}$, M. Dostál ${ }^{1,2}$, P. Kubát ${ }^{1}$, V. Nevrlý' ${ }^{2}$, P. Bitala ${ }^{2}$, and S. Civiš ${ }^{1}$ \\ ${ }^{1}$ J. Heyrovský Institute of Physical Chemistry AS CR, Prague, Czech Republic \\ ${ }^{2}$ Faculty of Safety Engineering, VŠB - Technical University of Ostrava, Ostrava, Czech Republic
}

Correspondence to: Z. Zelinger (zelinger@jh-inst.cas.cz)

Received: 24 July 2014 - Revised: 26 January 2015 - Accepted: 1 February 2015 - Published: 5 March 2015

\begin{abstract}
Laser photoacoustic spectroscopy (PAS) is a method that utilizes the sensing of the pressure waves that emerge upon the absorption of radiation by absorbing species. The use of the conventional electret microphone as a pressure sensor has already reached its limit, and a new type of microphone - an optical microphone - has been suggested to increase the sensitivity of this method. The movement of a micro-lever or a membrane is sensed via a reflected beam of light, which falls onto a position-sensing detector. The use of one micro-lever as a pressure sensor in the form of a silicon cantilever has already enhanced the sensitivity of laser PAS.

Herein, we test two types of home-made sensing elements - four coupled silicon micro-levers and a multilayer graphene membrane - which have the potential to enhance this sensitivity further. Graphene sheets possess outstanding electromechanical properties and demonstrate impressive sensitivity as mass detectors. Their mechanical properties make them suitable for use as micro-/nano-levers or membranes, which could function as extremely sensitive pressure sensors.

Graphene sheets were prepared from multilayer graphene through the micromechanical cleavage of basal plane highly ordered pyrolytic graphite. Multilayer graphene sheets (thickness $\sim 10^{2} \mathrm{~nm}$ ) were then mounted on an additional glass window in a cuvette for PAS. The movements of the sheets induced by acoustic waves were measured using an $\mathrm{He}-\mathrm{Ne}$ laser beam reflected from the sheets onto a quadrant detector. A discretely tunable $\mathrm{CO}_{2}$ laser was used as the source of radiation energy for the laser PAS experiments. Sensitivity testing of the investigated sensing elements was performed with the aid of concentration standards and a mixing arrangement in a flow regime. The combination of sensitive microphones and micromechanical/nanomechanical elements with laser techniques offers a method for the study and development of new, reliable and highly sensitive chemical sensing systems. To our knowledge, we have produced the first demonstration of the feasibility of using four coupled silicon micro-levers and graphene membranes in an optical microphone for PAS. Although the sensitivity thus far remains inferior to that of the commercial electret microphone (with an $S / N$ ratio that is 5 times lower), further improvement is expected to be achieved by adjusting the micro-levers and membrane elements, the photoacoustic system and the position detector.
\end{abstract}

\section{Introduction}

The objective of this paper lies in the context of new sensing technologies based on micromechanical sensing elements, including functional materials for gas sensing. These elements could be employed in the laser photoacoustic spectroscopy (PAS) method as part of a sensitive optical microphone. This technique offers several advantages compared with conventional spectroscopy; some of its major benefits include the fact that the detected signal is directly proportional to the laser intensity, the elimination of false absorption resulting from scattered light and the lack of a need for photodetectors, which perform poorly in the mid-infrared region.

PAS is a spectroscopic method that differs from other absorption spectroscopic techniques in the manner in which the absorbed radiation is detected. The absorbed light is con- 
verted into heat, which leads to gas expansion. If the excitation light is modulated or chopped, then the resulting pressure waves can be sensed by a microphone. Although conventional condenser microphones have reached their limits of sensitivity, the development of new pressure sensors offers an opportunity to increase the sensitivity of this technique.

It has been proposed that a cantilever-type pressure sensor be used in PAS in place of microphones to achieve optimal sensitivity (Kauppinen et al., 2004; Wilcken and Kauppinen, 2003; Kuusela and Kauppinen, 2007; Koskinen et al., 2008, 2006). The primary benefits of a cantilever are the very low string constant and the extremely wide dynamical range that can be achieved in the cantilever movement. The string constant can be 2 or 3 orders of magnitude smaller than that of the membrane of a condenser microphone, and the movement of the cantilever can span tens of micrometres without suffering any non-linear or restricting effects. A non-contact (deflection, interferometric) measurement of cantilever movement is required to avoid any damping caused by the probe and to maintain the wide dynamic range ( $\mathrm{Li}$ et al., 2012).

Micromechanical sensors represent a new branch of chemical sensing that utilizes a microfabricated spring (cantilever), as originally applied in atomic force microscopes (AFMs), for the recognition of interfacial mass- and charge-transfer processes with very high sensitivity. A detection system based on cantilever micromechanical behaviour already exists and is identical to that employed in AFMs (Jalili and Laxminarayana, 2004). This system has allowed the detection of analytes in concentrations down to the picomolar and sub-picomolar levels to become feasible in a variety of processes, including deposition/dissolution, adsorption/desorption ( $\mathrm{Ji}$ et al., 2001), solution $\mathrm{pH}$ changes and surface-confined charge-transfer reactions (Tabard-Cossa et al., 2005). For detection, both bending- and frequencyreadout micro-lever sensors can be employed (Battiston et al., 2001). Further development will be focused on attempts to construct cantilever arrays (Lang et al., 2005) modified by various receptors to increase the selectivity of the sensor response (Grogan et al., 2002) and to gain the ability to perform multicomponent analysis in a single step. The major advantages of microcantilever array sensors are their micrometrescale size, high sensitivity and short response time (Berger et al., 1997).

A good approximation to the ideal cantilever - i.e. a one-dimensional single nanocrystal - can be found in multi-/single-layer graphite crystals - i.e. multi-/single-layer graphene (MLG, SLG) (Novoselov et al., 2004). Graphene sheets possess outstanding electromechanical properties (Geim and Novoselov, 2007; Lee et al., 2008; Castro Neto et al., 2009) and demonstrate impressive sensitivity as mass detectors (Chen et al., 2009; Avdoshenko et al., 2012). Because of its highly uniform (if defect-free) structure, relatively high chemical stability, and outstanding mechanical properties such as a high Young's modulus and a low specific weight, which allow it to reach a high differential mass ratio and hence a high sensor sensitivity, graphene represents almost the ideal material for nanomechanical sensors. Nanomechanical vibrations have been investigated for potential application in nanothermometers (Rahmat et al., 2010).

In previous studies (to improve the physical modelling of urban air pollution), we have developed and employed laser photoacoustic spectrometry (Zelinger et al., 2004, 2006, 2009). The objective of this paper is to present the design and fabrication of several home-made sensing elements of the cantilever-and-membrane type and to test and characterize their mechanical properties with the aid of the developed PAS method. Transducers composed of various materials (silicon, carbon) were employed in the design of new gas-sensing elements.

The purpose of experiments that involve microphones, graphene-based membranes and silicon cantilevers is to examine the differences among various sensors to optimize the range of their utilization. Unlike microphones, cantilevers allow for detection in both frequency and resonance modes in addition to the deflection mode. In the case of a multilayer graphene membrane detector, we have now proven the feasibility of its use. This detector represents a fusion of a microphone membrane and a cantilever, the deflection of which is probed by a reflected laser beam. Its prospective advantage lies in the possibility of tuning its sensitivity by decreasing its stiffness. This can be accomplished by decreasing the number of graphene layers, which will also cause a shift in the resonant frequency. Most importantly, however, our approach includes an examination of a multicantilever/multimode set-up, which offers variability in both the mechanical properties and detection modes of the cantilevers and thus the choice of the detector with the most suitable response for a given application. There is also the possibility of receiving signals from a multidetector system and thus of improving the signal-to-noise ratio and minimizing detector failure. Both features are important for prospective utilization in detector units and for increasing automatic sensor-network coverage.

\section{Experimental}

A discretely tunable home-made $\mathrm{CO}_{2}$ laser emitting at rotation-vibration transitions of $\mathrm{CO}_{2}$ in the bands $\Sigma_{\mathrm{u}}^{+}\left(00^{0} 1\right)$ $-\Sigma_{\mathrm{g}}^{+}\left(10^{0} 0\right)$ and $\Sigma_{\mathrm{u}}^{+}\left(00^{0} 1\right)-\Sigma_{\mathrm{g}}^{+}\left(02^{0} 0\right)$ in a spectral range of 9-11 $\mu \mathrm{m}$ was used as the source of radiation energy for laser photoacoustic spectroscopy (PAS). The photoacoustic (PA) cell was designed as a cylindrical cell of $300 \mathrm{~mm}$ in length and $6 \mathrm{~mm}$ in diameter equipped with a microphone as well as either a graphene membrane or a cantilever sensor, which could be exchanged with each other or for a different membrane or cantilever. All sensor units were placed in the middle of the longitudinal dimension of the cell. The total internal volume of the PA cell was $19.5 \mathrm{~cm}^{3}$. To obtain the largest possible PA signal, it is necessary that the interior space of 
the PA cell, which is not exposed to laser radiation, is minimized. This means that in the case of a cylindrical PA cell, the inner diameter of the PA cell must be minimized with respect to the laser beam. The diameter of the laser beam that was used was approximately $9-10 \mathrm{~mm}$, and the laser beam was focused by a lens with a $25 \mathrm{~cm}$ focus onto the centre of the PA cell; this set-up motivated the geometry of the PA cell described above.

The sensitivity levels of the sensing elements were tested using concentration standards based on the permeation method (Okeeffe and Ortman, 1966; Barratt, 1981). The concentration standards were prepared in the form of closed tubes composed of permeable material (silicon, polyethylene, Teflon), and these standards were placed in a thermostatted chamber at $35^{\circ} \mathrm{C}$ for long-term weighing and storage. For measurements, each concentration standard was placed in a chamber through which carrier air was flowing. Using such a mixing arrangement, we were able to prepare a given concentration level in a flow regime (flow rate $1 \mathrm{~cm}^{3} \mathrm{~s}^{-1}$ ) and under atmospheric pressure, with methanol vapour as the testing gas. The $\mathrm{CO}_{2}$ laser was tuned to the 9 $\mathrm{P}(34) \mathrm{CO}_{2}$ laser line $\left(1033.488 \mathrm{~cm}^{-1}\right)$, which corresponds to the fundamental CO stretching band of methanol, where the maximal absorption cross section is $72 \times 10^{-20} \mathrm{~cm}^{2}$ (Loper et al., 1980).

We studied several AFM-based silicon cantilevers and graphene sheets via laser PAS. The membranes for the photoacoustic detector - graphene sheets - were prepared from MLG through micromechanical cleavage of basal plane Highly Ordered Pyrolytic Graphite (HOPG, ZYH grade, Bruker, USA) (Novoselov et al., 2004). Graphene sheets in the form of circular membranes (Fig. 1a) and AFMbased silicon cantilevers in a square arrangement (Fig. 1b) were tested. The MLG sheets (thickness $\approx 10^{2} \mathrm{~nm}$ ) were mounted on an additional glass window in the cuvette for PAS (Fig. 1a). The movements of the graphene sheets or the cantilever ends that were induced by acoustic waves were measured by an $\mathrm{He}-\mathrm{Ne}$ laser beam that was reflected from the sheets or the ends onto a quadrant detector (a redenhanced quad-cell silicon photodiode, SD 085-23-21-021, Laser Components). The signals from both the microphone and the quadrant detector were processed by an oscilloscope (LeCroy 9361).

Two types of cantilevers and one type of membrane were used: commercial (Bruker) silicon cantilevers of the OTESPA (metallized) type or the NP (metallized) type, with an arm length of $200 \mu \mathrm{m}$ and with resonant frequencies of $300 \mathrm{kHz}$ in ambient gas and $10 \mathrm{kHz}$ in liquid, and home-made multilayer graphene membranes. The primary benefits of using layered graphene as a membrane lie in its high elasticity (YM $\sim 1 \mathrm{TPa}$, defect free), the possibility of modifying its stiffness by simply removing graphene layers (down to the level of a single monolayer) and thus increasing its bending sensitivity, and, finally, its reflectivity, which allows the bending of the membrane to be probed using a reflected laser

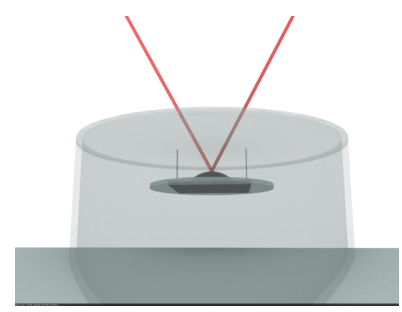

a)
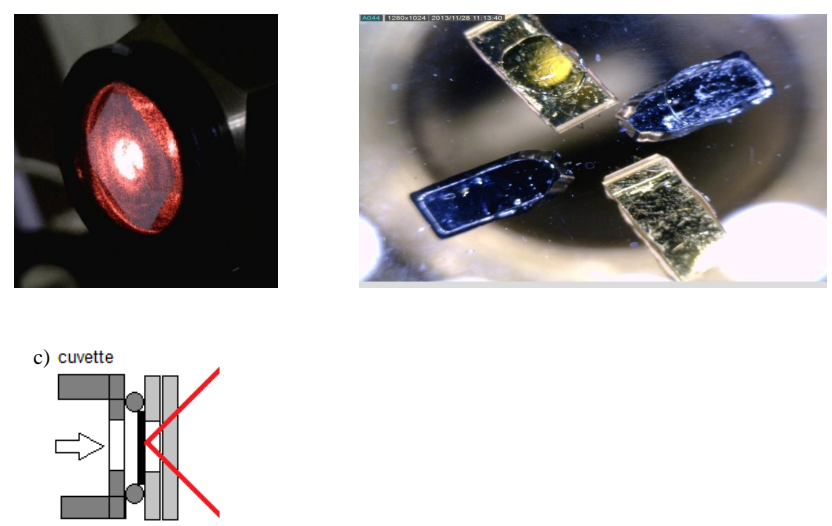

Figure 1. Schematic illustrations of the construction of the sensing elements and their implementations: (a) graphene-based sensing elements, (b) AFM-cantilever-based sensing elements, and (c) schematic illustration of the graphene membrane detector assembly on the cuvette window sealed with a silicone O-ring, where the red lines represent the laser beam and the arrow indicates the pressure wave.

beam. Compared with microphone membranes, cantilevers and graphene membranes exhibit lower damping and lower stiffness, and, therefore, higher sensitivity in general can be achieved. The graphene was of grade ZYH (Bruker, USA). The thickness of the multilayer graphene (MLG) could be simply determined through an AFM profile measurement. The thickness of the graphene that was used in these experiments was typically in the range of $10^{2} \mathrm{~nm}$, although this thickness could be further decreased.

The graphene membrane was attached to the glass window by an epoxy glue film and was pressed by an O-ring over the circular opening through which the pressure wave from the cuvette was transferred to the membrane. The membrane deflection was measured using a laser beam reflected from the membrane onto the quadrant detector. Details regarding the membrane mounting are provided in Fig. 1c.

The micromechanical sensing elements were installed in the cuvette for PAS together with a sensitive microphone (electret microphone EK 23024, Farnell) for comparison. The experimental set-up is depicted in Fig. 2. Quantitative measurements were performed using a phase-sensitive lockin amplifier (Stanford Research Systems SR530 lock-in amplifier). The $\mathrm{CO}_{2}$ laser beam was modulated by a chopper, and the photoacoustic signals from the microphone and the 


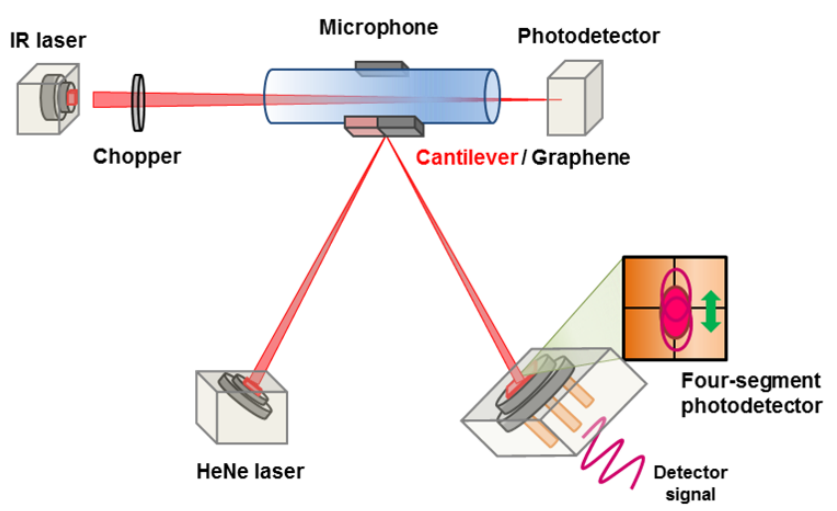

Figure 2. Experimental set-up for the tests of the sensing elements.

graphene were demodulated by the lock-in amplifier and subsequently processed using a PC. The signals from the microphone and from the cantilevers or graphene membrane were passed through preamplifiers to lock-in amplifiers. The reference signal from the chopper, i.e. the frequency of interruptions of the laser beam, served as a reference signal for the lock-in amplifiers. The input signal amplitude for the lockin amplifiers was $3 \mathrm{mV}$ for the microphone and $5 \mathrm{mV}$ for the cantilever and graphene sensors.

\section{Results and discussion}

AFM-based silicon cantilevers and MLG sheets were tested as photoacoustic detectors in these studies. Figure 1 shows a schematic diagram of the set-up for the testing of the MLGbased and silicon-AFM-cantilever-based sensing elements (henceforth referred to as graphene sensors and cantilever sensors, respectively, for brevity). In the case of a classical capacitive microphone, there is a space both in front of and behind the membrane that is connected by a balancing channel to eliminate any damping of the membrane's movement during its return. The same function is also performed in our sensing elements. The free ends of the cantilevers in the cantilever sensors can move freely in space. In the case of the graphene sensors, an additional glass slide is used as a specialized holder for the MLG-sheet-based membrane (Fig. 1a). The elimination of damping effects is proven by the experimental data presented in Fig. 3a. Figure 3a presents the motion of the MLG sheet as indicated by the $\mathrm{He}-\mathrm{Ne}$ laser beam reflected onto the quadrant detector, which translates the movement into a voltage amplitude proportional to the pressure changes inside the chamber. The low-frequency ( $4 \mathrm{~Hz}$ ) modulation of the laser by the chopper causes symmetrical movements of the graphene membrane.

The experimental data presented in Fig. 3a demonstrate the detection of the motion of the graphene sensor in the form of rising and falling exponentials. These observations are the result of the absorption of radiation, which heats the gas in the chamber. By virtue of the fixed volume, the time-varying thermal fluctuations generate pressure variations (sound waves), which are detected by the graphene sensor. The following conditions correspond to the typical case and are assumed to apply: the source is modulated such that the time profile approximates a square wave of period $T$; the molecular radiative and collisional relaxation times are small and can be neglected in comparison with the period of the chopper modulation $T$; and the detector chamber is a cylinder of length $l$ and radius $a$, where $l \gg a$. Under these conditions, it can be theoretically derived that the time dependence of the pressure response $p(\mathrm{t})$ is given in the following forms:

$$
\begin{array}{ll}
p(t)=c_{1}+c_{2}\left(1-e^{-t / \tau}\right) & 0 \leq t \leq T / 2, \\
p(t)=c_{2} e^{T / 2 \tau} e^{-t / \tau} & T / 2 \leq t \leq T,
\end{array}
$$

where $\tau$ is the thermal relaxation time to the temperature of the chamber walls and

$$
c_{1}=\Delta p \frac{\left(e^{-T / 2 \tau}-1\right)}{\left(e^{-T / 2 \tau}-e^{T / 2 \tau}\right)}, \quad c_{2}=\Delta p \frac{\left(1-e^{-T / 2 \tau}\right)}{\left(e^{-T / 2 \tau}-e^{T / 2 \tau}\right)},
$$

where $\Delta p$ corresponds to the fully developed pressure amplitude $(T \rightarrow \infty)$.

A gradual change from an exponential waveform to a sine waveform occurs as the modulation frequency is increased, at a frequency of approximately $23 \mathrm{~Hz}$ (Fig. 3b). Such waveforms are suitable for processing using a phase-sensitive amplifier (lock-in amplifier), thus allowing us to compare the sensitivities of the microphone and the sensing elements. The detection chamber was equipped with both the microphone and the tested sensing element (graphene sheet or silicon cantilevers). Both signals - from the microphone and from the quadrant detector (representing the detection of the motion of the sensing element: the graphene sensor or the cantilever sensor) - were processed using lock-in amplifiers. As a testing gas, we used methanol vapour. The detection chamber was connected to the flow system for precise monitoring of the flow rate of the carrier air using mass flow meters and controllers (FMA Series, OMEGA). A sampling chamber, into which the concentration standards were inserted, was placed in the flow system just in front of the detection chamber. These concentration standards provided a calibration of the measurements based on the permeation method (Okeeffe and Ortman, 1966; Stellmack and Street, 1983; Zelinger et al., 1988) and the long-term weighing of the standards.

Equivalent measurements were performed for the cantilever sensors (Fig. 1b). Silicon cantilevers offer the advantage of better optical reflection compared with the diffuse reflection from the MLG-sheet-based sensing elements (Fig. 1a). These experiments directly followed the research work previously performed by Kaupinnen et al. (Kauppinen et al., 2004; Koskinen et al., 2006, 2008; Kuusela and Kauppinen, 2007).

Waveforms were simultaneously recorded to monitor the time dependence of the intensity of the laser radiation, the 

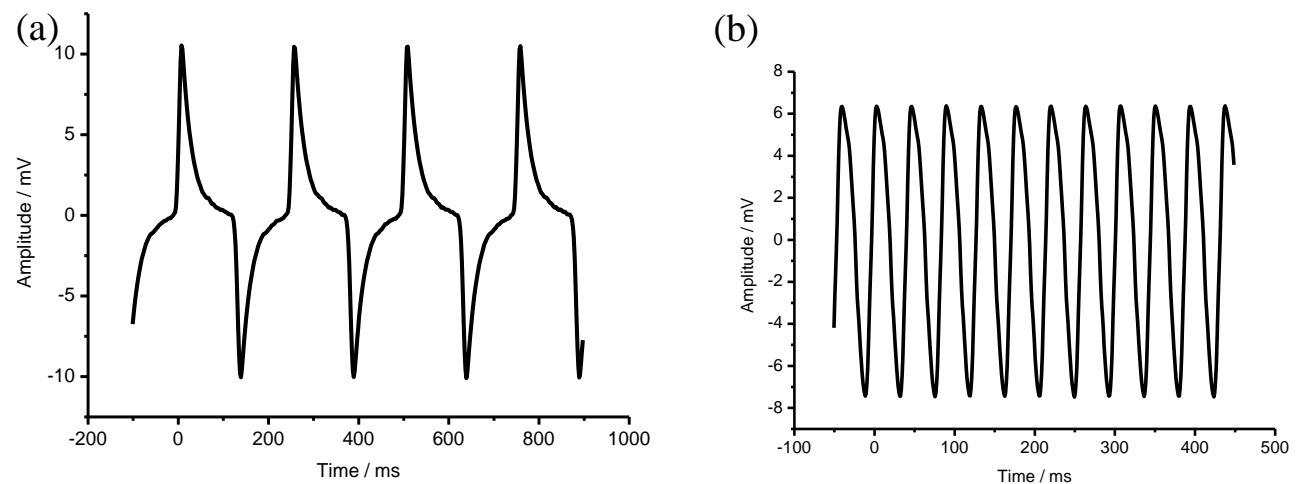

Figure 3. Experimental signals recorded from the graphene-membrane-based sensing elements at low frequency: (a) $4 \mathrm{~Hz}$ and (b) $23 \mathrm{~Hz}$.

time dependence of the response of the microphone as processed by the lock-in amplifier, and the time dependence of the response of the graphene sensor as processed by the lockin amplifier; the results are depicted in Fig. 4. The concentration of the methanol vapour that was introduced into the detection chamber was $\sim 10 \mathrm{ppm}$. The following steps were performed during each 500 s scan: the laser was initially off $(\mathrm{OFF})$ - the laser was turned on $(\mathrm{ON})$ - the laser was turned off (OFF) - the laser was turned on (ON) - the laser was turned off (OFF). Both the microphone and the graphene sensor responded to the turning on and off of the laser (Fig. 4). A rise and fall in the signals from both sensors can be observed upon the powering on and off of the laser, respectively, in Fig. 4; the magnitude of the difference in the signal is determined predominantly by the concentration of the absorbing gas, i.e. $10 \mathrm{ppm}$ of methanol. The background signal level (the difference between the signals recorded in the $\mathrm{ON}$ and OFF states of the laser at zero concentration of the absorbing gas) is almost negligible; in the case of the microphone, it is approximately twice as high as the noise level, and in the case of the cantilever, it is approximately half of the noise level.

In addition to the $\mathrm{ON}-\mathrm{OFF}$ mode of the laser, we also tested a similar ON-OFF mode for the concentration standard (Fig. 5). When we inserted a concentration standard ( 2 ppm) into the sample chamber (concentration ON), both the signal from the microphone and that from the graphene sensor (as processed by the lock-in amplifier) increased; see Fig. 5a. The same measurement was performed using the microphone and cantilever system; see Fig. 5b. Then, we removed the concentration standard from the flow system (concentration OFF) and monitored the resulting decrease in the signal (Fig. 5c, d). The responses of the microphone and graphene sensor are shown in Fig. 5c, and the responses of the microphone and cantilever system are shown in Fig. 5d. The perturbations observed at the beginning of the increase in the concentration signal (Fig. 5a, b) and at the beginning of the decrease in the concentration signal (Fig. 5c, d) were caused by pressure disturbances created in the sample cham-

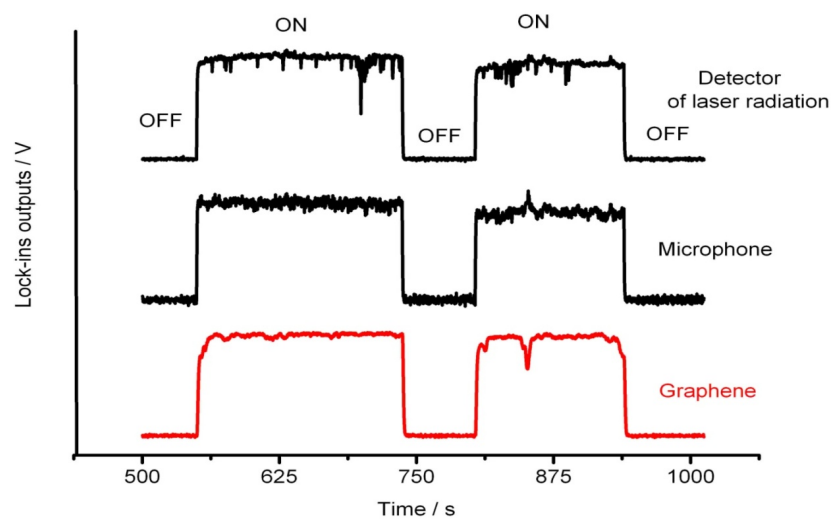

Figure 4. Experimental data for comparison of the signals detected by the microphone and by the graphene-based membranes at the same gas concentration (methanol, $\sim 10 \mathrm{ppm}$ ), as processed by the lock-in amplifiers.

ber when the concentration standard was inserted and removed.

A comparison of the noise levels indicated by the experimental data presented in Fig. 5 yields the corresponding signal-to-noise ratios $(S / N)$. The graphene sensors and the cantilever systems have different connections to the PA cell. The connection is in the middle of the cell and thus exerts a strong influence on the acoustic properties of the interior of the cell. In the connection of the graphene sensors or the cantilever systems to the PA cell, different regions are created that affect the acoustic signal gain, giving rise to a difference in the resulting sensitivities of the two systems. In the case of the microphone and the graphene sensor, the signal-tonoise ratio for the microphone was $S / N \approx 25$ and that for the graphene sensor was $S / N \approx 5$. In the case of the microphone and the cantilever system, the signal-to-noise ratio for the microphone was $S / N \approx 50$ and that for the cantilever system was $S / N \approx 36$. It is observed that the signal detected by the graphene sensor suffered from a higher noise level than did the signal from the cantilever. Possible reasons for this find- 
(a)

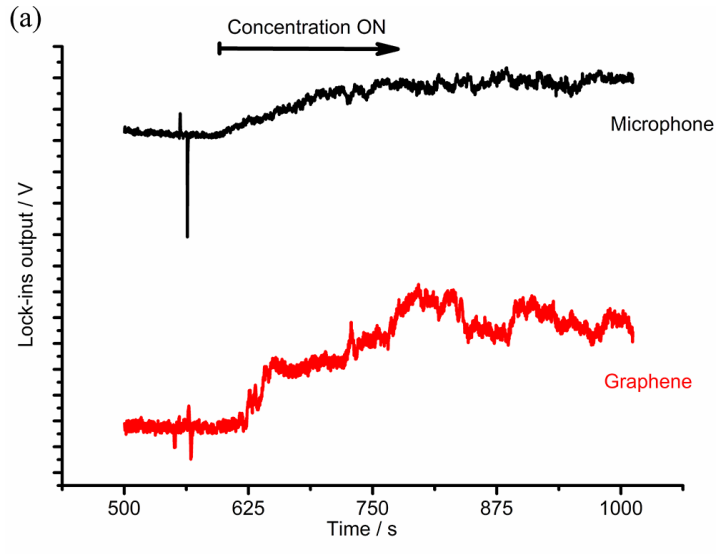

(c)

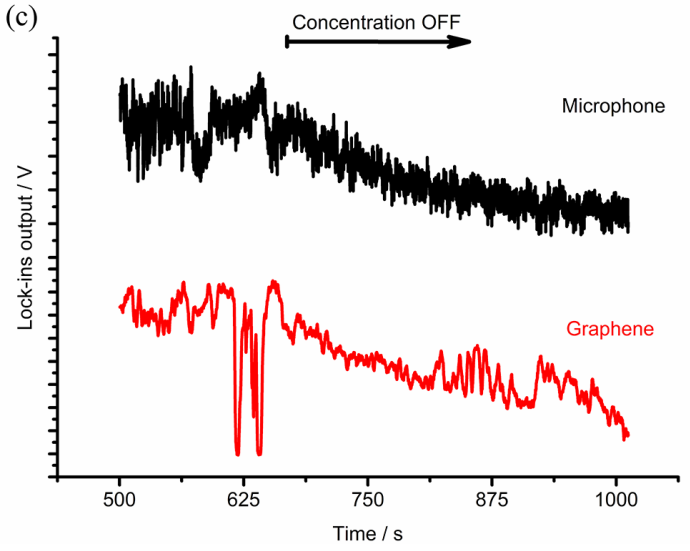

(b)

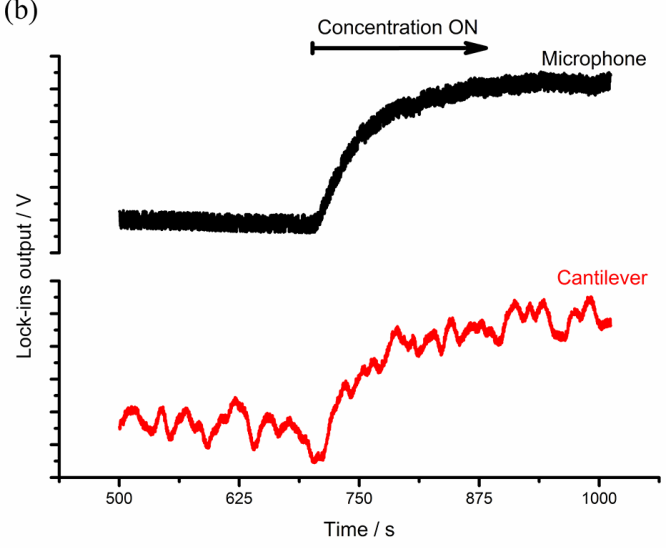

(d)

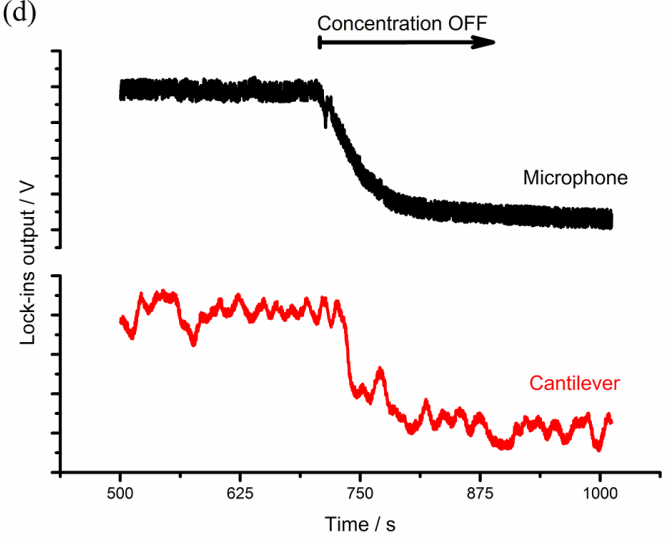

Figure 5. Experimental signals recorded from the lock-in amplifiers for a generated concentration of methanol of $\sim 2$ ppm: (a) concentration ON for the microphone and graphene sensor, (b) concentration ON for the microphone and cantilever system, (c) concentration OFF for the microphone and graphene sensor, and (d) concentration OFF for the microphone and cantilever system.

ing could lie in the adsorption properties of graphene with respect to those of methanol vapour; this phenomenon will be the subject of further studies. The cantilever system is not as strongly affected by the adsorption properties of the gas, and, therefore, its sensitivity approaches that of the microphone.

Thus far, the sensitivity of these elements has been inferior to that of a commercial microphone. We expect that further enhancement of the sensitivity can be achieved by adjusting the elements in combination with the entire photoacoustic system. To determine the optimal responses of the membranes, graphene sheets of different thicknesses will be prepared, and the dimensions of the cuvette (and thus the resonant frequency) will be varied. Reduction of the diffusion of the reflected laser beam can be achieved by depositing a thin layer of metal onto the membranes. Future work will focus on few-layer graphene cantilevers/membranes and singlelayer graphene, which will be prepared using the chemical vapor deposition (CVD) technique and then transferred to supports, thus allowing for free-standing mounting.

\section{Conclusions}

The first experimental results of analytical testing of MLGsheet-based sensing elements as well as results obtained for AFM-based silicon cantilever systems are presented. A method for the comparison of the responses of various sensing systems, including graphene sensors, cantilever systems and microphones, has been developed. We obtained the first quantitative data regarding the sensitivity of the tested graphene sensors and cantilever systems by applying concentration standards to generate concentrations of methanol in a well-defined way. The combination of sensitive microphones and micromechanical elements with advanced laser techniques offers possible opportunities for the development of new, reliable and highly sensitive chemical sensing systems.

Acknowledgements. The authors are grateful for the financial support provided via project no. LD14022 within the framework of COST action TD 1105 funded by the Ministry of Education, Youth and Sports of the Czech Republic, via project no. 14-14696S 
funded by the Grant Agency of the Czech Republic and via project no. R200401401 in the framework of the regional cooperation of the Academy of Sciences of the Czech Republic.

Edited by: A. L. Spetz

Reviewed by: three anonymous referees

\section{References}

Avdoshenko, S. M., Gomes Da Rocha, C., and Cuniberti, G.: Nanoscale ear drum: Graphene based nanoscale sensors, Nanoscale, 4, 3168-3174, doi:10.1039/c2nr30097d, 2012.

Barratt, R. S.: The preparation of standard gas mixtures a review, The Analyst, 106, 817-849, 1981.

Battiston, F. M., Ramseyer, J. P., Lang, H. P., Baller, M. K., Gerber, C., Gimzewski, J. K., Meyer, E., and Güntherodt, H. J.: A chemical sensor based on a microfabricated cantilever array with simultaneous resonance-frequency and bending readout, Sens Actuators, B. Chem., 77, 122-131, doi:10.1016/s09254005(01)00683-9, 2001.

Berger, R., Gerber, C., Lang, H. P., and Gimzewski, J. K.: Micromechanics: A toolbox for femtoscale science: "Towards a laboratory on a tip", Microelectron. Eng., 35, 373-379, 1997.

Castro Neto, A. H., Guinea, F., Peres, N. M. R., Novoselov, K. S., and Geim, A. K.: The electronic properties of graphene, Rev. Mod. Phys., 81, 109-162, doi:10.1103/RevModPhys.81.109, 2009.

Chen, C., Rosenblatt, S., Bolotin, K. I., Kalb, W., Kim, P., Kymissis, I., Sormer, H. L., Heinz, T. F., and Hone, J.: Performance of monolayer graphene nanomechanical resonators with electrical readout, Nat. Nanotechnol., 4, 861-867, doi:10.1038/nnano.2009.267, 2009.

Geim, A. K. and Novoselov, K. S.: The rise of graphene, Nat. Mater., 6, 183-191, doi:10.1038/nmat1849, 2007

Grogan, C., Raiteri, R., O’Connor, G. M., Glynn, T. J., Cunningham, V., Kane, M., Charlton, M., and Leech, D.: Characterisation of an antibody coated microcantilever as a potential immuno-based biosensor, Biosens. Bioelectron., 17, 201-207, doi:10.1016/s0956-5663(01)00276-7, 2002.

Jalili, N. and Laxminarayana, K.: A review of atomic force microscopy imaging systems: Application to molecular metrology and biological sciences, Mechatronics, 14, 907-945, doi:10.1016/j.mechatronics.2004.04.005, 2004.

Ji, H. F., Hansen, K. M., Hu, Z., and Thundat, T.: Detection of $\mathrm{pH}$ variation using modified microcantilever sensors, Sens Actuators, B. Chem., 72, 233-238, doi:10.1016/s09254005(00)00678-x, 2001.

Kauppinen, J., Wilcken, K., Kauppinen, I., and Koskinen, V.: High sensitivity in gas analysis with photoacoustic detection, Microchem. J., 76, 151-159, doi:10.1016/j.microc.2003.11.007, 2004.

Koskinen, V., Fonsen, J., Kauppinen, J., and Kauppinen, I.: Extremely sensitive trace gas analysis with modern photoacoustic spectroscopy, Vib. Spectrosc., 42, 239-242, doi:10.1016/j.vibspec.2006.05.018, 2006.
Koskinen, V., Fonsen, J., Roth, K., and Kauppinen, J.: Progress in cantilever enhanced photoacoustic spectroscopy, Vib. Spectrosc., 48, 16-21, doi:10.1016/j.vibspec.2008.01.013, 2008.

Kuusela, T. and Kauppinen, J.: Photoacoustic gas analysis using interferometric cantilever microphone, Appl. Spectrosc. Rev., 42, 443-474, doi:10.1080/00102200701421755, 2007.

Lang, H. P., Hegner, M., and Gerber, C.: Cantilever array sensors, Mater. Today, 8, 30-36, doi:10.1016/s1369-7021(05)00792-3, 2005.

Lee, C., Wei, X., Kysar, J. W., and Hone, J.: Measurement of the elastic properties and intrinsic strength of monolayer graphene, Science, 321, 385-388, doi:10.1126/science.1157996, 2008.

Li, P., You, Z., and Cui, T.: Graphene cantilever beams for nano switches, Appl. Phys. Lett., 101, 093111, doi:10.1063/1.4738891, 2012.

Loper, G. L., Calloway, A. R., Stamps, M. A., and Gelbwachs, J. A.: Carbon dioxide laser absorption spectra and low ppb photoacoustic detection of hydrazine fuels, Appl. Opt., 19, 2726-2734, 1980.

Novoselov, K. S., Geim, A. K., Morozov, S. V., Jiang, D., Zhang, Y., Dubonos, S. V., Grigorieva, I. V., and Firsov, A. A.: Electric field in atomically thin carbon films, Science, 306, 666-669, doi:10.1126/science.1102896, 2004.

Okeeffe, A. E. and Ortman, G. C.: Primary standards for trace gas analysis, Anal. Chem., 38, 760-763, doi:10.1021/ac60238a022, 1966.

Rahmat, F., Thamwattana, N., and Hill, J. M.: Carbon nanotube oscillators for applications as nanothermometers, J. Phys. Math. Theor., 43, 405209-405209 doi:10.1088/17518113/43/40/405209, 2010.

Stellmack, M. L. and Street, K. W.: Permeation devices for highpressure gases, Anal. Lett. Pt. A, 16, 77-100, 1983.

Tabard-Cossa, V., Godin, M., Beaulieu, L. Y., and Grütter, P.: A differential microcantilever-based system for measuring surface stress changes induced by electrochemical reactions, Sens Actuators, B. Chem., 107, 233-241, doi:10.1016/j.snb.2004.10.007, 2005.

Wilcken, K. and Kauppinen, J.: Optimization of a Microphone for Photoacoustic Spectroscopy, Appl. Spectrosc., 57, 1087-1092, doi:10.1366/00037020360695946, 2003.

Zelinger, Z., Papouskova, Z., Jakoubkova, M., and Engst, P.: Determination of trace quantities of freon by laser optoacoustic detection and classical infrared-spectroscopy, Collect. Czech. Chem. Commun., 53, 749-755, 1988.

Zelinger, Z., Střižík, M., Kubát, P., Jaňour, Z., Berger, P., Černý, A., and Engst, P.: Laser remote sensing and photoacoustic spectrometry applied in air pollution investigation, Opt. Lasers Eng., 42, 403-412, doi:10.1016/j.optlaseng.2004.03.005, 2004.

Zelinger, Z., Střižík, M., Kubát, P., Lang, K., Bezpalcová, K., and Jaňour, Z.: Model and real pollutant dispersion: Concentration studies by conventional analytics and by laser spectrometry, Int. J. Environ. An. Ch., 86, 889-903, 2006.

Zelinger, Z., Střižík, M., Kubát, P., Civis, S., Grigorová, E., Janečková, R., Zavila, O., Nevrlý, V., Herecova, L., Bailleux, S., Horká, V., Ferus, M., Skřínský, J., Kozubková, M., Drábková, S., and Jaňour, Z.: Dispersion of light and heavy pollutants in urban scale models: co 2 laser photoacoustic studies, Appl. Spectrosc., 63, 430-436, doi:10.1366/000370209787944226, 2009. 